\title{
A Longitudinal Study on International Students' Stress, Problem Focused Coping and Cross-Cultural Adaptation in China 来华留学生跨文化压力应对和适应研究 ${ }^{1}$
}

\author{
Alexander English 英亚东 \\ Shanghai International Studies University, China \\ 上海外国语大学，中国 \\ Ruobing Chi 迟若冰 \\ Shanghai International Studies University, China \\ 上海外国语大学，中国
}

\begin{abstract}
Academic research conducted in the west has shown that people in individualistic and collectivist cultures cope with cross-cultural adaptation stress differently, but there is still a lack of understanding how coping may function in the nonWestern context. We conducted a longitudinal study to explore how the relationship between perceived cultural distance, stress- and problem-focused coping strategies may influence socio-cultural adaptation for international students in China. We collected longitudinal data on overseas students during the Fall semester of September 2013. Data were recollected 90 days later. The results show that: (1) perceived cultural distance did not predict the outcome of socio-cultural adaptation; (2) non-Asian students used more problem-focused coping strategies than Asian students; (3) interaction effect between stress, coping strategy and culture of origin is significant: for non-Asian students, problem-focused coping strategies alleviated the negative impact of stress on adaptation; and for Asian students, problem-focused coping strategies exacerbated the negative impact of stress on adaptation. This paper concludes with interpretation of our findings and how these results can be useful in higher education institutions in China.
\end{abstract}

摘要:有研究显示个体和集体主义文化背景下，人们应对跨文化适应压力的方式 不同，但目前还缺乏在非西方背景下对相关结论的验证。本研究以来华留学生为

\footnotetext{
${ }^{1}$ 本研究与第一作者在 SpringerPlus 上发表的文章（English, Zeng, \& Ma, 2015) 属于同一纵向研究项 目，因此部分内容有重合，特此声明。
} 
研究对象, 采用纵向设计, 探究了文化距离、压力和问题应对策略与社会文化适 应之间的关系。研究对象为 121 名在华留学生 (亚洲 52 人, 非亚洲 69 人), 数 据收集间隔为 90 天。研究发现: (1) 感知的文化距离不能预测社会文化适应结果; (2) 与亚洲留学生相比, 非亚洲留学生更多使用问题中心应对策略; (3) 压力、应 对策略和种族的交互效应显著: 对非亚洲留学生, 问题中心应对策略能够缓解压 力对于适应的消极影响; 而对亚洲留学生, 问题中心应对策略加剧了压力对于适 应的消极影响。

Keywords: acculturative stress, cultural distance, problem-focused coping, crosscultural adaptation 关键词: 跨文化适应, 文化距离, 社会文化适应, 问题中心应 对策略

自 2010 年中国实施《留学中国计划》后, 来华留学生规模快速增长。2015 年, 共有 202 个国家和地区的近 40 万名留学生在中国学习, 比 2010 年增加了 $50 \%$ （中国教育部, 2017）。2017 年 3 月初教育部在新闻发布会上公布的 2016 年留学 生数据显示, 中国已经成为亚洲重要的留学目的国。每年来自欧美的留学生约有 6 万，亚洲和非洲的学生数量则更为庞大（Bodomo, 2014）。2018 年共有来自 196 个国家和地区的 492,185 名各类外国留学人员在中国 31 个省（自治区、市） 的 1004 所高校学习, 比 2017 年增加了 3,013 人, 增长 $0.62 \%$ (以上数据均不含 港、澳、台地区留学生）（English, Allison, \& Ma, 2016; Hashim \& Yang, 2003）。

高等教育的国际化和全球化推动了来华留学人数的快速增长, 留学生的文化 适应和管理问题也随之产生, 受到中国高等教育界的重视。跨文化适应受众多因 素影响, 相关研究角度多样。已有不少研究探讨了性格特点、语言能力、学习动 力、跨文化交往等与留学生在华社会文化适应和心理健康之间关系 (An \& Chiang, 2015; Fu \& Gan, 2004; Liu \& Jia, 2009; Qi \& Li, 2009; Yu, 2010），以及对 留学生跨文化适应模式的分析和总结 (An, 2009; Lu, 2000)。本文希望在前人的 基础上, 对留学生群体作进一步细分和比较, 以留学生普遍需要应对的多种压力 为切入点, 对文化距离假设 (Babiker, Cox, \& Miller, 1980) 和压力-应对-适应模 型（Berry, 2005; Lazarus \& Folkman, 1984）在中国社会文化环境中的适用性进行 实证检验。

研究探讨的主要问题是：不同地区来华留学生的跨文化适应是否有差异? 与 中国文化较接近的群体是否适应得更好? 来华留学生面对跨文化适应压力时是否 采用了问题中心的应对策略? 这一策略对压力和适应是否具有调节作用? 此外, 跨文化适应的时间跨度较大, 纵向实证检验的研究方法更有助于揭示各因素之间 的相关关系 (Selmer, 2006; Ward, 2004) , 对该领域常见的横向实证研究取向也 是有益的补充（Kuo, 2014），因此本文采用纵向研究设计。

\section{相关文献综述与研究假设}

\section{感知文化距离}

Babiker 等人（1980）指出文化距离（即母国文化与现居地文化之间的差异） 决定了跨文化适应的难度。后续大量研究支持了这一假设, 即文化距离越大, 社 
会文化适应和心理适应的结果越差（Galchenko \& van de Vijver, 2007; Ward \& Kennedy, 1999）。这些研究主要采用 GDP 等综合经济指标或文化价值观指数等 集体层面的变量来衡量文化距离, 然而旅居者本身对文化距离的感知并不一定与 此一致。因此有必要对感知文化距离和跨文化适应结果之间的关系做进一步探索。

Nesdale 和 Mak (2003) 对感知文化距离的研究结论与文化距离假设相同, 他们发现来自新西兰的学生在澳大利亚感知到的文化差异最小, 遇到的心理问题 也最少。东亚学生群体感受到更多的文化差异, 他们的跨文化适应结果也更差。 然而 Forster（1997）的研究发现在英国的美国人感觉到的文化差异不大, 但是遇 到的适应困难与来自差异性大的文化背景的旅居者没有什么不同。Selmer（2006） 的研究发现在中国的西方旅居者虽然感受到明显的文化差异, 但是相比没有感受 到明显差异的香港人，他们适应的反而更好。可见，感知文化距离是否和文化距 离一样对跨文化适应起作用还没有定论, 因此本研究将其作为第一个假设进行检 验:

$\mathrm{H} 1$ ：感知文化距离与社会文化适应结果相关。

\section{跨文化适应压力}

无论是真实存在的还是感知到的文化差异, 都可能导致旅居者在生活、工作 和学习中面临诸多困难, 感到压力重重。Smart和 Smart（1995）将人们进入新的 文化环境后感受到的困难定义为跨文化适应压力。这种压力可能源自文化环境、 人际交往、学习任务或心理因素。例如, 在中国大学的非洲留学生在参与社会活 动方面感到困难（Hashim \& Yang, 2003）。

Berry（2005）认为跨文化适应压力根植于适应过程，因不同的旅居目的而 不同（如留学生和移民所面临的压力不同）。实证研究显示旅居者面对的压力会 对心理健康和社会文化适应产生负面影响（Mähönen \& Jasinskaja-Lahti, 2013; Rudmin, 2009; Wei et al., 2007）。而国际学生除了和其它旅居者受到一样的压力 外, 还会面临学习压力, 语言困难和社会支持缺乏等困难（Wei et al., 2007）, 因此我们认为对留学生而言:

$\mathrm{H} 2$ ：跨文化压力与社会文化适应结果呈负相关。

\section{问题中心应对策略}

既然起负面作用的跨文化压力无法避免，那么有效的应对策略就成为跨文化 适应的关键。Lazarus 和 Folkman（1984）及 Carver（1989）开启了压力应对策略 的研究。他们将应对策略分为两种: 问题中心策略和情绪中心策略。问题中心策 略着眼于压力源, 个体采取直接的行动去解决压力事件。情绪中心策略着眼于个 体情绪, 通过调节或改变自身对压力源的认知和感受来应对压力。Szabo 等人

（2017）在其关于中国和新西兰的国际留学生跨文化适应对比研究中, 详细探讨 了亚洲和非亚洲学生在情绪中心应对策略使用上的差别, 以及该策略对其所受跨 文化压力和适应结果的影响。但是对于亚洲和非亚洲学生在问题中心应对策略使 用上是否有所区别, 以及该策略对跨文化压力和适应的作用还不明确, 因此本研 究选择聚焦于此, 采用平行设计, 探讨相同的问题, 以便对之前研究有所补充。 
研究显示不同种族、移民及难民群体都可以通过采取问题中心策略来控制和 应对心理压力（Folkman et al., 1986）。比如 Ward 和 Kennedy（2001）对居住在 新加坡的英国人的调查发现, 问题中心策略能够预测较低的抑郁水平。但是对社 会文化适应方面，问题中心策略是否同样有效还不明确。本研究预测:

H3：问题中心策略与社会文化适应结果呈正相关。

对不同文化背景下应对策略的探究多是关注不同种族群体应对压力方式的差 异 (Jose \& Huntsinger, 2005; Jose \& Schurer, 2010; Selmer, 2002; Sinha \& Watson, 2007）。一般认为个人主义文化背景下的个体更愿意采用问题中心策略, 集体主 义文化背景下的个体则较倾向于使用情绪中心策略（Chun et al., 2006）。比如, Lam 和 Zane (2004) 发现亚裔美国人比美国白种人更多使用情绪中心策略。 Spector 等人 (2004) 发现, 与美国学生相比, 香港学生更多使用情绪中心应对 策略。Selmer（2002）的研究显示在中国的西方人比亚洲人更多使用问题中心策 略。本研究对此作出相同预测, 即:

H4：与非亚洲国家来华留学生相比, 亚洲国家来华留学生更多使用问题中 心应对策略（PFC）。

但是研究者也指出问题中心策略的使用并不一定说明它是有效的（Aldwin, 2007; Chun et al., 2006; Heppner, 2008; Wong, Wong, \& Scott, 2006）。Cervantes 和 Castro 在 1985 年提出压力-中介-适应模型。该模型认为压力是通过应对策略中介 对适应产生作用。而 Aldwin（2007）指出, 应对策略也可以是一个调节变量, 与压力形成交互作用, 继而影响适应的结果。比如 Crockett 等（2007）以及 Noh 和 Kaspa（2003）的研究都发现, 问题中心策略可以有效消除压力对于心理适应 的负面影响。也有研究显示应对策略对压力-适应所起的调节作用因种族而异, 其影响 (加剧还是减轻) 需要具体问题具体分析 (Cohen \& Wills, 1985; Jose \& Huntsinger, 2005; Jose, Ward, \& Liu, 2007; Yoo \& lee, 2005）。因此, 有研究者呼 吁更多针对不同种族群体的实证研究来验证此类调节作用（Kuo, 2014; Wilson, Ward, \& Fischer, 2013）。这也是本研究希望探讨的最后一个假设:

H5: 问题中心策略和种族的交互关系对压力-适应具有调节作用。

综上所述, 本研究希望揭示不同种族在华留学生群体在感知文化距离、跨文 化适应压力、应对策略上的差异，以及这些因素对他们社会文化适应结果的影响。

\section{研究方法}

\section{研究对象}

共有 121 名来华留学生参与本研究。他们来自 24 个不同的国家和地区。基 于 Galchenko 和 van de Vijver (2007) 的群体划分方法, 本研究根据地理位置以 及感知文化距离的远近将研究对象分为两组: 第 1 组为亚洲国家来华留学生, 共 有 52 人, 包括东亚 (日本, 韩国) 和东南亚国家 (泰国, 越南, 印度尼西亚, 马来西亚) 的学生。第 2 组为非亚洲国家来华留学生, 共有 69 人, 包括欧洲 (意 大利，法国，德国，英国）、北美（加拿大，美国）和非洲一些国家的学生 ${ }^{\text {。 }}$ 具体情况见表 1 。

\footnotetext{
${ }^{1}$ 来自非洲的学生人数较少 $(\mathrm{n}=17)$, 且他们的感知文化距离测量结果与欧美来华留学生无明显差异, 故合并为一组。
} 
表 1. 研究对象概况

\begin{tabular}{lcccccc}
\hline \multirow{2}{*}{ 项目 } & \multicolumn{2}{c}{ 亚洲 $(\mathrm{n}=52)$} & \multicolumn{2}{c}{ 非亚洲 $(\mathrm{n}=69)$} & \multicolumn{2}{c}{ 合计 $(\mathrm{N}=121)$} \\
\cline { 2 - 6 } & 平均 & 标准差 & 平均 & 标准差 & 平均 & 标准差 \\
\hline 居住时间 $\mathrm{a}$ & 199 & 345 & 112 & 175 & 148 & 261 \\
年龄 & 24.91 & 7.2 & 21.85 & 3.27 & 23.13 & 5.5 \\
性别 & 男: 23 & $46 \%$ & 男: 34 & $46 \%$ & 男: 57 & $49 \%$ \\
& 女: 29 & $54 \%$ & 女: 35 & $54 \%$ & 女: 64 & $51 \%$ \\
中文水平 b & 初级 & 31 & 初级 & 42 & 初级 & 73 \\
& 中级 & 17 & 中级 & 19 & 中级 & 36 \\
& 高级 & 4 & 高级 & 8 & 高级 & 12 \\
\hline
\end{tabular}

注. ${ }^{\mathrm{a}}$ 居住时间以天为单位。 ${ }^{\mathrm{b}}$ 中文水平为自我评价的结果。

\section{问卷}

问卷包括感知文化距离、压力、问题中心策略和社会文化适应四个量表。

\section{感知文化距离}

采用 Galchenko 和 van de Vijver（2007）的感知文化距离量表。量表由 22 道 测试题组成, 采用 7 点计分, 1 表示非常不同, 7 表示非常相似。本研究中该量 表的内部一致性系数为 0.90 。

\section{压力}

采用 Jose, Cafasso 和 D’Anna（1994）的压力测试量表。该量表包含 36 道测 试题, 要求被试根据最近一个月的情况作答。问卷采用 4 点计分, 0 表示没有压 力, 3 表示压力很大。测量内容包括沟通、饮食、出行、气候、健康等方面的压 力。其中一道与英语口音有关的题由于与中国环境不符而删除。本研究中该量表 的内部一致性系数为 0.88 。

\section{问题中心策略}

采用 Carver, Scheier 和 Weintraub（1989）的应对策略量表。该量表包括 8 道 测试题, 分别测量行动和计划两个方面, 采用 4 点计分, 1 表示完全不会这样做, 4 表示经常这样做。本研究中该量表的内部一致性系数为 0.84 。

\section{社会文化适应}

采用 Wilson, Ward, Fetvadjiev 和 Bethel（2017）的社会文化适应量表修订版。 该量表包括 21 道测试题。量表采用 5 点计分法, 1 表示不能适应, 5 表示非常适 应。本研究中该量表的内部一致性系数为 0.88 。 


\section{研究过程}

本研究的问卷有英文、中文、韩文 ${ }^{1}$ 三种版本, 被试可根据自己的实际情况 选择不同语言的问卷。完成问卷需 20-30 分钟。

被试为中国东部二线城市四所学校的留学生。问卷在他们的中文课以及中国 概况课课堂上发放。数据采集分两次进行。第一次为 2014 年 9 月初, 即被试初 到中国学习的第一周。90 天后, 研究者在第一次发放问卷的课堂发放第二次测 试量表。问卷使用被试学生证的前四位及后四位数字作为编码, 对两次数据进行 匹配。整个研究过程中被试自愿参加, 并有自主权可以随时退出。

\section{统计方法}

采用 spss 20.0 进行数据整理和分析。

\section{结果分析}

\section{相关分析}

相关分析的结果见表 2 。由表 2 可知, 感知文化距离与社会文化适应结果不 相关, 假设 1 检验结果不成立。跨文化压力与留学生在中国的居住时间和他们的 年龄都相关, 但是与社会文化适应结果不相关, 假设 2 检验结果不成立。问题中 心策略与社会文化适应呈正相关, 假设 3 检验结果成立。

表 2. 相关分析结果

\begin{tabular}{lccccccc}
\hline 变量 & 1 & 2 & 3 & 4 & 5 & 6 & 7 \\
\hline 1. 中文 & - & & & & & & \\
2. 在华时间 & 0.13 & - & & & & & \\
3. 年龄 & 0.01 & 0.16 & - & & & & \\
4. 感知文化距离 & 0.02 & 0.00 & 0.10 & - & & \\
5. 压力 & -0.09 & $0.21 *$ & $-0.19^{*}$ & -0.15 & - & & \\
6. 问题中心策略 & -0.08 & -0.04 & -0.04 & $0.25 * *$ & $-0.24 * *$ & - & \\
7. 社会文化适应 time 2 & 0.13 & 0.01 & $-0.29 * *$ & 0.07 & -0.08 & $0.20^{*}$ & - \\
\hline
\end{tabular}

注. $* p<.05$ （2-tailed）;** $p<.01 （ 2$-tailed）;*** $p<.001 （ 2$-tailed）。

\section{组别比较}

表 3 列出了组别比较结果。从中可见, 不同族群使用问题中心策略存在显著 差异 $(\mathrm{F}(1,120)=6.19, p=0.014, \eta 2=0.05)$, 假设 4 得到验证。他们的社会文化适 应也存在显著差异 $(F(1,120)=10.67, p=0.001, \eta 22=0.08 ） 。$

\footnotetext{
1 中国教育部公布的数据显示韩国多年来一直居于来华留学生来源国榜首, 我们的被试中韩国留学生 也占多数, 因此提供韩语版问卷。
} 


\section{表 3. 组别比较结果}

\begin{tabular}{|c|c|c|c|c|c|c|}
\hline \multirow{2}{*}{ 量表 } & \multicolumn{2}{|c|}{ 亚洲 } & \multicolumn{2}{|c|}{ 非亚洲 } & \multicolumn{2}{|c|}{ 全体 } \\
\hline & M & SD & M & SD & M & SD \\
\hline 感知文化距离 & 4.96 & 0.83 & $5.38 * *$ & 0.57 & 5.20 & 0.72 \\
\hline 问题中心策略 & 2.86 & 0.54 & $3.12 * *$ & 0.53 & 3.01 & 0.54 \\
\hline 压力 & 18.31, & 10.49 & 16.35 & 5.88 & 17.19 & 8.20 \\
\hline 社会文化适应 & 3.01 & 0.62 & $3.37 * * *$ & 0.62 & 3.21 & 0.63 \\
\hline
\end{tabular}

注: $* * p<0.01, * * * p<0.001$

本研究数据还显示, 男女留学生在感知文化距离、应对策略和跨文化压力方 面有显著差异。男性感知到的文化距离比女性更大 $(\mathrm{Mm}=5.45, \mathrm{SD}=0.72 \mathrm{vs}$. $\mathrm{Mf}=4.97, \mathrm{SD}=0.64$ ），也更多使用问题中心策略（ $\mathrm{Mm}=3.19 \mathrm{SD}=0.49$ vs. $\mathrm{Mf}=2.84$ $\mathrm{SD}=0.54$ ）。女性感到的压力更大（ $\mathrm{Mf}=18.82 ， \mathrm{SD}=8.72$ vs. $\mathrm{Mm}=15.35$, $\mathrm{SD}=7.22$ ）。因此, 在多元回归分析中将性别作为控制变量（Yoo \& Lee, 2005）。

\section{表 4. 多元回归分析结果}

\begin{tabular}{lcc}
\hline \multirow{2}{*}{ 自变量 } & \multicolumn{2}{c}{ 因变量: 社会文化适应 } \\
\cline { 2 - 3 } 第一步 & Beta & \multicolumn{1}{|c}{$\mathrm{R}^{2} / \Delta \mathrm{R}^{2}$} \\
\hline 年龄 & $-0.26^{* *}$ & \\
性别 & 0.01 & \\
在华时间 & -0.05 & \\
中文 & 0.12 & \\
种族 & $-0.22^{*}$ & $0.17 / 0.01$ \\
第二步 & & $0.19 / 0.01$ \\
压力 & -0.10 & \\
第三步 & & $0.26 . / 0.07 * *$ \\
问题中心策略 & 0.12 & \\
第四步 & & \\
问题中心策略 $\times$ 压力 & $0.24^{*}$ & \\
问题中心策略 $\times$ 种族 & 0.03 & $0.30 / 0.04 * *$ \\
压力 $\times$ 种族 & -0.19 & \\
第五步 & & \\
\hline 问题中心策略 $\times$ 压力 $\times$ 种族 & $-0.46^{* * *}$ & \\
\hline
\end{tabular}

注: 种族 $(0=$ 非亚洲人, $1=$ 亚洲人 $)$, 性别（ $0=$ 女, 男 $=1 ）$ 。

${ }^{*} p<0.05 ; * * p<0.01 ; * * * p<0.001$ 。 


\section{多元回归分析}

对假设 5 的检验采用了分层多元回归分析方法。具体借鉴了 Aiken,West \& Reno（1991）的三因素交互效应分析方法。第一步, 将人口学变量 (年龄, 性 别, 居住时间, 种族）加入回归方程; 第二、三步, 加入压力和问题中心策略变 量; 第四步, 加入压力 $\times$ 问题中心策略, 压力 $\times$ 种族, 问题中心策略 $\times$ 种族双因 素交互项; 最后, 加入压力 $\times$ 种族 $\times$ 问题中心策略三因素交互项。为了减少共线 性, 对所有自变量、调节变量都进行了中心化处理（Fock et al., 2013）。表 4 列 出了分析结果。从中可见, 压力 $\times$ 种族 $\times$ 问题中心策略的交互项对留学生社会文 化适应具有显著影响 $\left(R^{2}=0.30, b=-0.46, p=0.01\right)$ 。

接着采用 Cohen \& Wills (1985) 和 Jose 等人 (2015) 的方法, 对交互效应 进行简单斜率分析, 并用 Modgraph（Jose, 2013）软件绘图对结果进行说明。

从图 1 和图 2 中可以看出, 对于亚洲留学生来说, 问题中心策略加剧了压力 对于社会文化适应的负面影响 $(t=-2.11, p=0.04)$ 。相反, 对于非亚洲留学生来 说, 问题中心策略缓解了压力对于社会文化适应的负面影响, 有利于他们的社会 文化适应 $(t=3.09, p<0.01)$ 。

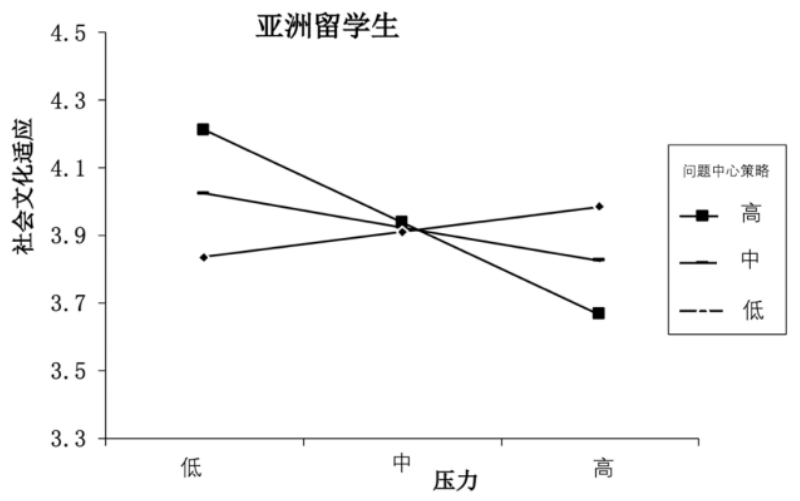

图 1. 问题中心策略在压力与社会文化适应之间的调节作用（亚洲群体）

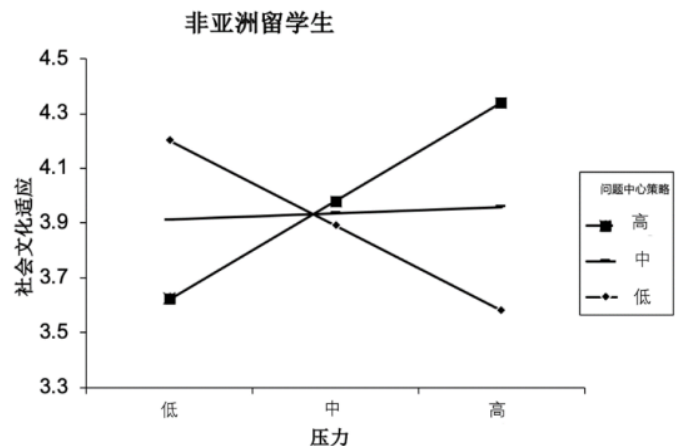

图 2. 问题中心策略在压力与社会文化适应之间的调节作用（非亚洲群体） 


\section{讨论}

本研究的主要目的是探索不同来源地的留学生在中国的跨文化适应情况及应对压 力方式的差异, 研究采用纵向设计方法, 对两个不同群体的跨文化适应压力、文 化距离、问题中心策略、社会文化适应之间的关系进行了实证检验。

近年来, 文化与语境应对模式将应对过程定义为个体与环境相互作用的结果。 当个体采用一致性的文化应对策略时，他们往往适应得更好。类似的 “文化契合” 理论研究也表明, 个体所采用的策略（例如应对策略）与其文化背景相关

（Heppner et al., 2014）。因此，采用文化上适当的策略能促进更成功的文化过渡。 近期证据表明有效的文化一致性应对对适应的影响（Ward \& Geeraert, 2016）。

Szabo 等人 (2017) 进行的一项纵向研究发现, 二级应对策略加剧了新西兰亚洲 和西方留学生文化适应压力的负面影响。相比之下, 对于在中国的亚洲和西方留 学生群体来说, 二级应对策略缓解了文化适应压力的负面影响。换句话说, 二级 应对策略代表了中国集体主义环境下的文化一致性应对策略，因此具有适应性， 而它却导致了新西兰个人主义背景下的文化错配。因此, 未来的研究应该着手分 析一致或不一致的应对策略是如何影响旅居者的适应的。

本研究发现感知文化距离的大小与留学生的社会文化适应不相关。数据显示 感知文化距离仅与问题中心策略呈正相关关系, 即感知文化距离越大, 使用问题 中心策略的频率也越高。该结果支持了近年来对文化距离假设有效性和适用性的 批评（Selmer, 2002; Selmer, 2006）。因此, 还需在采用不同样本对感知文化距离 的影响进行更深入的研究。

与预测相反, 本研究并未发现跨文化压力与适应结果相关。原因可能是应对 策略在压力与跨文化适应结果之间起了调节作用（Aldwin, 2007）。从对假设 5 的对交互作用的检验结果看很可能是这样的。

本研究还发现, 与亚洲留学生相比, 非亚洲留学生更多使用问题中心策略。 这与 Chun et al. (2006) 、Heppner（2008）和 Selmer（2002）的研究结果一致, 也间接印证了 Berry（2005）的推断, 即某些应对策略更适合某些特定的跨文化 群体。由此可见, 不同文化背景的群体采用的应对策略存在差异, 个人主义社会 中的个体可能会更多使用问题中心策略。今后还需要对更多不同文化背景的群体 进行类似的检验, 以观察该结论的普适性。

本研究发展, 使用问题中心策略有利于留学生的社会文化适应。进一步的交 互作用分析发现，问题中心策略和种族能够调节压力一社会文化适应之间的关系。 问题中心策略仅对非亚洲留学生群体有效; 亚洲留学生更多使用问题中心策略反 而会加剧压力给他们的社会文化适应带来的负面影响, 降低其适应水平。这一结 果与 Kuo (2014) 的研究一致。Kuo 认为可能的原因是, 亚洲留学生在适应一个 亚洲文化背景的国家时, 为避免打破与环境的和谐平衡, 更倾向于使用回避或者 集体（主义）的应对策略，而非问题中心策略。

\section{创新及局限}

很多研究者（Chun et al., 2006; Kuo, 2013; Sinha \& Watson, 2007）认为, 目前关于 应对策略在压力一适应关系中所起的作用和影响还没有获得一致的结论, 需要针 
对不同文化背景中的不同群体进行更多更深入的研究。从全球视野来看, 中国在 国际化教育中发挥着越来越重要的作用, 来华留学生人数大幅度增加（Belyavina, 2013）。关于这些留学生在中国的跨文化适应问题变得更为重要。因此, 本研究 选择了来华留学生作为研究对象, 对他们感知的文化距离、跨文化压力、采用的 应对策略和社会文化适应的关系进行了实证检验。此外, 本研究采用的纵向设计 恰好与跨文化适应领域众多的横向研究形成互补, 使我们能更加全面地了解这一 过程中产生的种种变化（Berry, 2005）。

总的来说, 感知文化距离并不是适应过程中的关键因素, 其大小对预测社会 文化适应没有帮助。研究较好地解释了问题中心策略在跨文化适应过程中的作用: 对于某些特定的群体, 使用问题中心策略能够缓解压力带来的负面影响, 提高社 会文化适应程度, 而对于其他群体则不然。就实践意义而言, 本研究为中国高校 提供了留学生跨文化适应方面的实证依据, 有助于学校有针对性地为来自不同地 区的留学生提供咨询和帮助, 提高管理质量。研究结果对于亚洲其他国家以及在 世界范围内促进跨文化适应也有一定借鉴作用。

本研究也存在一定局限性。首先, 提供的问卷仅有三种语言选择 (中文、英 文、韩文），无法确保来自不同语言地区的被试能够对问卷具有基本相似的理解。 将来可以将问卷翻译成更多种的语言, 对更多的群体进行考察（Demes \& Geeraert, 2014）。

其次, 数据收集采用自我报告方式, 不同文化背景的被试可能会有不同的应 答倾向, 比如来自儒家文化背景的亚洲被试, 可能受和谐及中庸价值观的影响, 自我报告时与个人主义文化背景下的个体不完全一致。

再者, 本研究采用的量表均为西方学者所设计, 虽然有助于与其它国家所做 的相似研究进行比较, 但在本土适切性上的确有所局限。今后的研究者如果能加 入本土量表, 并辅以质性研究设计, 应当可以为理解来华留学生的跨文化适应提 供更具参考价值的结论。

最后, 本研究虽然采用了纵向设计, 但是时间跨度以自然学期长度为准, 只 有 90 天, 并不算长, 无法全面呈现跨文化适应全过程中压力和适应之间关系的 变化。

\section{参考文献}

Aiken, L. S., West, S. G., \& Reno, R. R. (1991). Multiple regression: Testing and interpreting interactions. Newbury Park, CA, US: Sage.

An, R., \& Chiang, S. Y. (2015). International students' culture learning and cultural adaptation in China.Journal of Multilingual and Multicultural Development,36(7), 661-676.

Aldwin, C. M. (2007).Stress, coping, and development: An integrative perspective. New York, NY, US: Guilford Press.

Babiker, I. E., Cox, J. L., \& Miller, P. M. (1980). The measurement of cultural distance and its relationship to medical consultations, symptomatology and examination performance of overseas students at Edinburgh University.Social Psychiatry,15(3), $109-116$.

Belyavina, R. (2013). US students in China: Meeting the goals of the 100,000 strong initiative. New York, NY: Institute of International Education. 
Berry, J. W. (2005). Acculturation: Living successfully in two cultures.International journal of Intercultural Relations,29(6), 697-712.

Bodomo, A. (2014). The African traveller and the Chinese customs official: Ethnic minority profiling at border check points in Hong Kong and China? Journal of African American Studies, 1-13.

Carver, C. S., Scheier, M. F., \& Weintraub, J. K. (1989). Assessing coping strategies theoretically based approach. Journal of Personality and Social Psychology, 56(2), 267-283.

Cervantes, R. C., \& Castro, F. G. (1985). Stress, coping, and Mexican American mental health: A systematic review.Hispanic Journal of Behavioral Sciences,7(1), 1-73.

Cohen, S., \& Wills, T. A. (1985). Stress, social support, and the buffering hypothesis.Psychological Bulletin, 98(2), 310-357.

Chun, C. A., Moos, R. H., \& Cronkite, R. C. (2006). Culture: A fundamental context for thestress and coping paradigm. In P. T. P. Wong \& L. C. J. Wong (Eds.), Handbook of multicultural perspectives on stress and coping (pp. 29-53). Springer.

Crockett, L. J., Iturbide, M. I., Torres Stone, R. A., McGinley, M., Raffaelli, M., \& Carlo, G. (2007). Acculturative stress, social support, and coping: Relations to psychological adjustment among Mexican American college students.Cultural Diversity and Ethnic Minority Psychology, 13(4), 347-355.

Demes, K. A., \& Geeraert, N. (2015). The highs and lows of a cultural transition: A longitudinal analysis of sojourner stress and adaptation across 50 countries.Journal of Personality and Social Psychology, 109(2), 316-337.

English, A. S., Zeng, Z. J., \& Ma, J. H. (2015). The stress of studying in China: primary and secondary coping interaction effects. SpringerPlus, 4(1), 755. https://doi.org/10.1186/s40064-015-1540-3

English, A. S., Allison, J., \& Ma, J. H. (2016). Understanding Western students: Motivations and benefits for studying in China. Journal of Education and Training Studies, 4(8), 44-55.

Forster, N. (1997). The persistent myth of high expatriate failure rates: A reappraisal. International Journal of Human Resource Management, 8(4), 414-433.

Folkman, S., Lazarus, R. S., Dunkel-Schetter, C., DeLongis, A., \& Gruen, R. J. (1986). Dynamics of a stressful encounter: cognitive appraisal, coping, and encounter outcomes. Journal of Personality and Social Psychology, 50(5), 992-1003.

Fock, H., Hui, M. K., Au, K., \& Bond, M. H. (2013). Moderation effects of power distance on the relationship between types of empowerment and employee satisfaction. Journal of Cross-Cultural Psychology, 44(2), 281-298.

Galchenko, I., \& van de Vijver, F. J. (2007). The role of perceived cultural distance in the acculturation of exchange students in Russia. International Journal of Intercultural Relations, 31(2), 181-197.

Hashim, I. H., \& Zhiliang, Y. (2003). Cultural and gender differences in perceiving stressors: A cross-cultural investigation of African and Western students in Chinese colleges. Stress and Health, 19(4), 217-225.

Heppner, P. P. (2008). Expanding the conceptualization and measurement of applied problem solving and coping: From stages to dimensions to the almost forgotten cultural context. American Psychologist, 68, 805-816.

Heppner, P. P., Weisz, J. R., Neville, H. A., \& Kanagui-Mu-oz, M. (2014). A cultural and contextual model of coping. In F. T. L. Leong, L. Comas-Díaz, G. C. Nagayama Hall, V. C. McLoyd, \& J. E. Trimble (Eds.), APA handbook of 
multicultural psychology, Vol. 2: Applications and training. APA handbooks in psychology (pp. 83-106). Washington, DC, US: American Psychological Association.

Jose, P \& Huntsinger, C. (2005) Moderation and mediation effects of coping by Chinese American and European American adolescents, The Journal of Genetic Psychology, 166(1), 16-44.

Jose, P. E., \& Schurer, K. (2010). Cultural differences in coping among New Zealand adolescents. Journal of Cross-Cultural Psychology, 41(1), 3-18.

Jose, P., Ward, C., \& Liu, J. H. (2007). Cross-cultural adaptation of Asian and Western international students in New Zealand. Casting the Individual in Societal and Cultural Contexts, 205-224.

Jose, P. E., Cafasso, L. L., \& D’Anna, C. A. (1994). Ethnic group differences in children's coping strategies. Sociological Studies of Children, 6, 25-53.

Jose, P. E. (2013). ModGraph-I: A program to compute cell means for the graphical display of moderational analyses. Wellington, New Zealand: Victoria University of Wellington.

Kuo, B. C. (2014). Coping, acculturation, and psychological adaptation among migrants: A theoretical and empirical review and synthesis of the literature. Health Psychology and Behavioral Medicine: An Open Access Journal, 2(1), 16-33.

Lam, A. G., \& Zane, N. W. (2004). Ethnic differences in coping with interpersonal stressors: A test of self-construals as cultural mediators. Journal of Cross-Cultural Psychology, 35(4), 446-459.

Lazarus, R. S., \& Folkman, S. (1984). Stress, appraisal, and coping. New York, NY, US: Springer publishing company.

Mähönen, T. A., \& Jasinskaja-Lahti, I. (2013). Acculturation expectations and experiences as predictors of ethnic migrants' psychological well-being. Journal of Cross-Cultural Psychology, 44(5), 786-806.

Nesdale, D., \& Mak, A. S. (2003). Ethnic identification, self-esteem and immigrant psychological health. International Journal of Intercultural Relations, 27(1), 2340.

Noh, S., \& Kaspar, V. (2003). Perceived discrimination and depression: Moderating effects of coping, acculturation, and ethnic support. American Journal of Public Health, 93(2), 232-238.

Rudmin, F. (2009). Constructs, measurements and models of acculturation and acculturative stress. International Journal of Intercultural Relations, 33(2), 106123.

Selmer, J. (2006). Cultural novelty and adjustment: Western business expatriates in China.The International Journal of Human Resource Management,17(7), 12091222.

Selmer, J. (2002). Coping strategies applied by Western vs overseas Chinese business expatriates in China.International Journal of Human Resource Management,13(1), 19-34.

Sinha, B. K., \& Watson, D. C. (2007). Stress, coping and psychological illness: A crosscultural study. International Journal of Stress Management, 14(4), 386-397.

Smart, J. F., \& Smart, D. W. (1995). Acculturative stress of Hispanics: Loss and challenge. Journal of Counseling \& Development,73(4), 390-396.

Spector, P. E., Sanchez, J. I., Siu, O. L., Salgado, J., \& Ma, J. (2004). Eastern versus Western control beliefs at work: An investigation of secondary control, 
socioinstrumental control, and work locus of control in China and the US.Applied Psychology, 53(1), 38-60.

Szabo, A., English, A. S., Zhijia, Z., Jose, P., Ward, C., \& Jianhong, M. (2017). Is the utility of secondary coping a function of ethnicity or the context of reception? A longitudinal study across Western and Eastern cultures. Journal of Cross-Cultural Psychology,48(8), 1230-1246.

Ward, C., \& Geeraert, N. (2016). Advancing acculturation theory and research: The acculturation process in its ecological context. Current Opinion in Psychology, 8, 98-104.

Ward, C., \& Kennedy, A. (1999). The measurement of sociocultural adaptation. International Journal of Intercultural Relations, 23(4), 659-677.

Ward, C. (2004) Psychological theories of culture contact and their implications for intercultural training. In D. Landis, M. Bennett, \& J. Bennett (Eds.), Handbook of Intercultural Training (3rd ed., pp. 185-216). Thousand Oaks, CA, US: Sage.

Ward, C., \& Kennedy, A. (2001). Coping with cross-cultural transition. Journal of Cross-cultural Psychology, 32(5), 636-642.

Wei, M., Heppner, P. P., Mallen, M. J., Ku, T. Y., Liao, K. Y. H., \& Wu, T. F. (2007). Acculturative stress, perfectionism, years in the United States, and depression among Chinese international students. Journal of Counseling Psychology,54(4), 385-394.

Wilson, J., Ward, C., Fetvadjiev, V. H., \& Bethel, A. (2017). Measuring cultural competencies: The development and validation of a revised measure of sociocultural adaptation. Journal of Cross-Cultural Psychology,48(10), 14751506.

Wilson, J., Ward, C., \& Fischer, R. (2013). Beyond culture learning theory: What can personality tell us about cultural competence?. Journal of Cross-cultural Psychology,44(6), 900-927.

Yoo, H. C., \& Lee, R. M. (2005). Ethnic identity and approach-type coping as moderators of the racial discrimination/well-being relation in Asian Americans.Journal of Counseling Psychology, 52(4), 497-506.

Yu, B. (2010). Learning Chinese abroad: The role of language attitudes and motivation in the adaptation of international students in China.Journal of Multilingual and Multicultural Development, 31(3), 301-321.

安然. (2009). 来华留学生跨文化适应模式研究. 中国高等教育, (18), 61-62.

中华人民共和国教育部. (2017). 2016 年度我国来华留学生情况统计.Retrieved from http://www.moe.gov.cn/jyb_xwfb/xw_fbh/moe_2069/xwfbh_2017n/xwfb_17030 1/170301_sjtj/201703/t20170301_297677.html

雷龙云, \& 甘怡群. (2004). 来华留学生的跨文化适应状况调查. 中国心理卫生杂志, $18(10), 729-729$.

刘宏宇, \& 贾卓超. (2014). 来华留学生跨文化适应研究一以来华中亚留学生为个 案. 中央民族大学学报: 哲学社会科学版, 41(4), 171-176.

吕玉兰. (2000). 来华欧美留学生的文化适应问题调查与研究. 首都师范大学学报 (社会科学版) , (S3), 158-170.

元华, \& 李秀妍. (2009). 在京韩国留学生跨文化适应问题研究. 青年研究, (2), 84-93. 


\section{作者简介（Author biography）}

英亚东, 美国人, 浙江大学应用心理学博士, 上海外国语大学跨文化研究中心博 士后研究学者。电子邮箱: AlexEnglish@ @ shisu.edu.cn

ALEXANDER ENGLISH (USA, Zhejiang University, Phd in Applied Psychology) currently is a post-doc researcher at Shanghai Intercultural Institute at Shanghai International Studies University. Research interests are intercultural competence, crosscultural adaptation and internal migration. Email: AlexEnglish@shisu.edu.cn

迟若冰 (通讯作者), 美国夏威夷大学传播与信息科学博士, 上海外国语大学 跨文化研究中心副研究员。电子邮箱: crbcherry@ shisu.edu.cn

RUOBING CHI (corresponding author) obtained her Ph.D. at the University of Hawaii in 2014. She is an associate research fellow at the Intercultural Institute in Shanghai International Studies University. Her research lies in the interdisciplinary space between interpersonal/intergroup communication, social networks, and acculturation. Email: crbcherry@shisu.edu.cn 